\title{
El ocio y la recreación en las sociedades latinoamericanas actuales
}

\author{
Christianne L. Gomes ${ }^{1}$
}

UFMG/CNPq/FAPEMIG, Belo Horizonte, Brasil. Email: chrislucegomes@gmail.com

Resumen: ${ }^{2}$ El objetivo de este artículo es analizar el ocio y la recreación en las sociedades latinoamericanas actuales según el punto de vista de estudiosos de estas temáticas. La metodología involucró una investigación bibliográfica y entrevistas con 25 profesores, profesionales y estudiantes vinculados a cinco programas de maestría en el área de la recreación, el tiempo libre y el ocio que son realizados en cuatro países latinoamericanos: Brasil, Costa Rica, Ecuador y México. Las consideraciones de los entrevistados fueron muy diversas e indican la importancia de revisar la forma habitual de conceptualizar al ocio y a la recreación principalmente en función del trabajo. Este aspecto atravesó, de modo transversal, distintas entrevistas. La formación de profesionales para el campo de la recreación, el tiempo libre y el ocio necesita contemplar el desafío de pensar críticamente en el sentido de buscar alternativas para las graves problemáticas presentes en las sociedades latinoamericanas, algo que es cada vez más urgente y necesario.

Palabras clave: Ocio, recreación, América Latina.

\section{Recreation and leisure in current Latin American societies}

Abstract: The aim of this paper is to analyze the role of leisure and recreation in current Latin American societies from the point of view of specialists in these subjects. The methodology involved a literature review and interviews with 25 lecturers, professionals and students linked to five master programs in the area of recreation, free time and leisure located in four Latin American countries: Brazil, Costa Rica, Ecuador and Mexico. The considerations of the respondents were highly diverse and indicate the importance of reviewing the usual way of conceptualizing leisure and recreation mainly in relation to work. This aspect was present transversally in different interviews. The preparation of professionals in the field of recreation and leisure needs to consider the challenge of critical thinking in order to find alternatives to the serious social problems within Latin American societies, something that is becoming ever more urgent and necessary.

Key words: Leisure, recreation, Latin America.

\section{Recreação e lazer nas sociedades latino-americanas atuais}

Resumo: O objetivo deste artigo é analisar o lazer e a recreação nas sociedades latino-americanas atuais, segundo o ponto de vista de estudiosos dessas temáticas. A metodologia envolveu uma pesquisa bibliográfica e a realização de entrevistas com 25 professores, profissionais e estudantes vinculados a cinco programas de mestrado na área da recreação, tempo livre elazer que são realizados emquatro países latino-americanos: Brasil, Costa Rica, Equador e México. As considerações dos entrevistados foram muito diversas e indicam a importância de revisara forma 
habitual de conceituar o lazer e a recreação principalmente em função do trabalho. Este aspecto cruzou de modo transversal, distintas entrevistas. A formação de profissionais para o campo da recreação, tempo livre e lazer precisa contemplar o desafio de pensar criticamente no sentido de buscar alternativas para as graves problemáticas que se apresentamnas sociedades latino-americanas, algo que é cada vez mais urgente e necessário.

Palavras-chave: Lazer, recreação, América Latina.

\section{Introducción: Lineamientos teórico-metodológicos}

Diversos autores latinoamericanos apuntan a las dificultades conceptuales que rondan a las palabras ocio, recreación y términos similares, tales como animación sociocultural y tiempo libre, entre otros. Muchas veces esos términos son utilizados indistintamente, generando equívocos conceptuales, contradicciones y variados problemas de comprensión, como indica Elizalde (2010). De igual forma, es posible constatar una pluralidad de sentidos y significados atribuidos al ocio y la recreación en los países de América Latina, lo que pudiendo ser un incentivo para la ampliación de compresiones, en muchos casos, paradojalmente termina comprometiendo la profundización y avance de los conocimientos en este campo de estudios.

Siendo así, en este artículo se destaca la importancia del ocio, en cuanto una práctica social contextualizada, al ser comprendido y reconocido como una dimensión de la cultura históricamente situada que está en íntimo diálogo con el contexto y las realidades locales específicas. De esta manera, cada pueblo y cada sociedad construye y significa sus prácticas socioculturales de ocio y recreativas, que son vividas como disfrute de la cultura y de la vida social en sus propias temporalidades. Siguiendo esta línea de interpretación, se considera que una nueva mirada sobre el ocio precisa ser construida rescatando los saberes, experiencias y prácticas socioculturales existentes en cada territorio.

Desde aquí el ocio es comprendido como una necesidad humana y como una dimensión de la cultura caracterizada por la vivencia lúdica de manifestaciones culturales en el tiempo/espacio social. Las manifestaciones culturales que constituyen el ocio pueden ser consideradas prácticas sociales vividas como disfrute de la cultura (Gomes, 2011). Entendidas así, las diversas manifestaciones culturales de ocio son saberes, representaciones, expresiones y lenguajes humanos lúdicamente vivenciados, englobando también los instrumentos, objetos, artefactos y lugares culturales que integran la vida cotidiana de comunidades, grupos y personas.

Obviamente, las variadas manifestaciones culturales son posibles de transformación y son influenciadas por distintas sociedades y culturas, especialmente en un mundo globalizado. Por esto, el ocio no se refiere solamente a las prácticas llamadas tradicionales o folclóricas, sino también 
a todo tipo de práctica social generalmente considerada como moderna y emergente. Entre ellas, por ejemplo, pueden ser citadas las experiencias ligadas a la virtualidad, los nuevos gustos musicales, estilos de fiestas o determinadas actividades que para una persona de otra generación o de otra cultura pueden no ser comprendidos.

A su vez, se considera que el ocio es más amplio que la recreación. En ese sentido, al ser el ocio entendido como una necesidad humana, la recreación podrá ser una de las posibles formas de satisfacer la necesidad de ocio. Por esto, es importante comprender que el ocio puede incluir a la recreación, lo que no ocurre de forma inversa. Así, se considera que el ocio y la recreación, desde lo conceptual, son diferentes. (Gomes; Elizalde, 2012)

Además de esto, es posible percibir que el ocio y la recreación son prácticas sociales presentes en cada sociedad, lo que nos lleva a preguntar: ¿qué lugar ocupan el ocio y la recreación en las sociedades latinoamericanas actuales?

Antes de abordar esta pregunta, se considera importante destacar los marcos teóricos que fundamentan las reflexiones y los análisis que aquí serán desarrollados. Estos marcos están relacionados, primordialmente, con las teorías que buscan comprender los tiempos y espacios culturales de hoy, generalmente llamados de "postmodernos", según las proposiciones de Follari (2004).

Muchas dificultades e incomprensiones aparecen cuando se piensa en la denominación más adecuada para designar el momento histórico-social vivido actualmente. Por eso, se utilizan distintas nomenclaturas para definirlo, tales como "postmodernidad" (adoptada, por ejemplo, por autores como Harvey, 1993 y Jameson, 1999), “modernidad tardía” (Hall, 2005), "capitalismo tardío" (Sennet, 2006), "sociedad de hiperconsumo" (Lipovetsky, 2007) y “modernidad líquida” (Bauman, 2001), entre otras.

Aunque esas expresiones puedan involucrar distintas perspectivas y provocar mucha polémica, es posible afirmar que todas comparten tres aspectos comunes. El primero de ellos es que todas son parciales, como es propio de cualquier comprensión teórica -sobre todo en contextos tan dinámicos, cambiantes y heterogéneos como los actuales. Esta primera limitación genera un segundo aspecto en común: todas son posibles de críticas, lo que puede deberse no sólo a las preferencias y posturas conceptuales específicas, sino también a las diversificadas influencias del contexto social. El tercer aspecto se refiere al hecho de que todas estas perspectivas procuran comprender el momento presente y asumen, como punto de partida para sus análisis, los cambios ocurridos en el transcurso del siglo XX, especialmente a partir de su segunda mitad.

En este sentido, para este texto, se considera que más importante que la denominación a ser utilizada para designar el momento actual, lo determinante es el potencial aporte de un concepto o teoría para impulsar 
las reflexiones que se hacen necesarias para comprender el rol asumido por el ocio y la recreación en las sociedades contemporáneas.

Según Bittar (2008), la contemporaneidad es marcada por una gama de modificaciones quese proyectan sobre diversas dimensiones de la vida social. Como enfatiza la autora, obviamente este proceso envuelve valores, hábitos, acciones grupales, necesidades colectivas, reglas sociales, concepciones y variados modos de organización institucional, entre otros elementos que constituyen y conforman las subjetividades.

Siendo así, los múltiples cambios culturales, sociales, políticos y tecnológicos transcurridos en el siglo XX son, al mismo tiempo, producto del agotamiento de las referencias anteriores y del descubrimiento de nuevas posibilidades generadas por el avance técnico. Se trata, por lo tanto, de una lógica cultural propia del capitalismo tardío, que propicia los mecanismos que generan una subjetividad que se asemeja a la superficialidad fluctuante propia del zapping, de los videoclip, de los celulares, de la Internet, de los juegos y de las experiencias virtuales, de la exposición televisiva en busca de fama y de dinero. Todo esto genera en muchos casos una pérdida de los compromisos ideológicos y sociales colectivos, la erosión de la ética, la falta de crítica, la caída de las vanguardias y del valor subversivo del arte, que son reemplazados por la experiencia cotidiana del espectáculo mediático. Así, muchas reflexiones fueron instigadas por la existencia de espacios culturales y mediáticos denominados postmodernos que, como indica Follari (2004: 23), conforman las subjetividades:

Ante el aumento del tamaño de las ciudades, la velocidad que permiten los viajes, la desterritorialización de la experiencia por el mail, la TV cable y el internet (entre otros múltiples factores concurrentes), la subjetividad está modificándose radicalmente. Asistimos a sujetos móviles, con sostenimiento de creencias sin pregnancia, lábiles y cambiantes. Es decir, ya los valores se enraízan débilmente en los sujetos, pues estos mismos están débilmente constituidos, son atravesables cada vez por flujos nuevos que los redefinen y modifican permanentemente.

El autor anteriormente citado aclara que las modalidades de vida cotidiana contemporáneas son claramente distintas de aquellas que marcaron las llamadas sociedades modernas. Así, lo “postmoderno” fue una consecuencia de la consumación y agotamiento de la razón moderna, cuyas promesas de progreso continuo y de solución de los problemas humanos, a partir de la supremacía de la razón, manifiestan sus límites e insuficiencias. Por un lado, la primacía de la razón instrumental fue decisiva para la evolución tecnológica. No obstante, por otro lado, la racionalidad instrumental se ha revelado insuficiente para solucionar los grandes problemas sociales de países como aquellos que integran la América Latina, entre muchos otros países del mundo. Problemáticas que están marcadas por la pobreza, miseria, discriminación, exclusión, racismo, etc., mostrándose también ineficientes para promover la sustentabilidad socioambiental. 
Vista desde ese ángulo la postmodernidad se trata de una perspectiva que, según Follari (1990), es incompetente para permitir la preservación del medio ambiente e inútil para concebir un modelo político-económico democrático, eficiente y comprometido con la superación de las desigualdades sociales. Considerar estos y otros graves problemas que presentan las sociedades latinoamericanas, entre muchas otras, se destaca la importancia de comprender críticamente el momento presente para poder actuar sobre él (Follari; Lanz, 1998).

Boaventura de Sousa Santos (2011:23-24) complementa esta discusión al destacar, en relación a la promesa de igualdad, que los países capitalistas avanzados siendo el 21\% de la población mundial controlan el 78\% de la producción de bienes y servicios, y a su vez consumen el 75\% de toda la energía producida a nivel global. Paralelamente, las violaciones de los derechos humanos en países viviendo formalmente en paz y democracia asumen proporciones avasalladoras. Millones de niños trabajan en régimen de cautiverio y muchos otros viven en la calle. La acción policial y carcelaria es cada día más violenta y los incidentes raciales aumentan, junto a la agresión social contra mujeres y la prostitución infantil. A esto se suman las diversas formas de discriminación, tales como la ejercida contra toxicodependientes, portadores de VIH y homosexuales, además del fanatismo racial, étnico y religioso. Como resalta el autor, en el siglo XVIII murieron 4,4 millones de personas en 68 guerras y en el siglo XX murieron 99 millones de personas en 237 guerras. A su vez, enfatiza que en los últimos 50 años el mundo perdió cerca de 1/3 de su capa forestal y la desertificación y la falta de agua son uno de los problemas que más afectarán a los países del tercer mundo en las próximas décadas, ya que hoy en día 1/5 de la humanidad no tiene acceso al agua potable.

La comprensión de las causas de estas problemáticas y la búsqueda de alternativas para enfrentarlas está vinculada a un proceso de formación político-pedagógica que requiere profundizar críticamente sobre ellas. A pesar de lo anterior, es cada vez más común tratar estas temáticas de forma superficial, sensacionalista y efímera, logrando con esto que la población pierda su capacidad de indignarse frente a esta situación. Esto es agravado por la influencia de los medios masivos de comunicación, tales como la TV, y también por las nuevas tecnologías de la información y comunicación, que no necesariamente contribuyen en la generación de una visión crítica y cuestionadora de la realidad social actual.

Así, los nuevos medios desarrollados y popularizadas en varios países desde mediados de la década de 1990 pueden cumplir diferentes funciones: informativa, educativa, política, de interacción social, de diversión, etc. Pero en el cumplimiento de esas funciones los medios masivos van penetrando e interfiriendo en la acción de otras instituciones y en otros campos sociales, tales como la familia, la escuela, el trabajo, el ocio, etc.

En el contexto actual, otro aspecto a ser destacado es el que las nuevas tecnologías colaboran con la compresión del espacio-tiempo. Esta 
expresión, según Harvey (1993), significa que el tiempo parece acelerarse y las distancias parecen estar más cortas. Consecuentemente, el pasado y el futuro se disuelven, ocurriendo lo que el autor denomina de reinado del presente donde todo lugar está al alcance de un instante. Así, la compresión espacio-tiempo se refiere a los procesos que acaban por revolucionar las cualidades objetivas del espacio y del tiempo. De este modo, se cambia radicalmente la manera como cada persona representa el mundo para sí mismo, así como concibe y realiza las diferentes actividades cotidianas. Todo esto acaba moldeando comportamientos y configurando nuevas prácticas socioculturales, muchas de las cuales intensifican la comunicabilidad instantánea a través de medios tecnológicos, la valorización de la imagen, la mutabilidad, la pasividad, y paralelamente el individualismo, la evasión, la apatía y el consumismo.

Tomando en cuenta estas nuevas configuraciones culturales, sociales, políticas y educativas, entre otras que marcan la vida contemporánea en diferentes latitudes, ¿qué lugar ocupan hoy el ocio y la recreación en las sociedades latinoamericanas?

Como ya fue mencionado, esta fue la pregunta clave que guió el presente estudio, que es parte integrante de una investigación más amplia sobre la formación profesional y académica en el área del ocio y de la recreación en América Latina. Así, el objetivo de este artículo es analizar el papel del ocio y de la recreación en las sociedades latinoamericanas actuales, según el punto de vista de estudiosos de estas temáticas.

La metodología de esta investigación cualitativa (Laville, Dionne, 1999) combinó distintas estrategias: estudio bibliográfico, análisis documental y realización de entrevistas semi-estructuradas con 25 voluntarios. Los entrevistados son profesores, profesionales y estudiantes vinculados a cinco programas de maestría en el área de la recreación, el tiempo libre y el ocio ("lazer") que se realizan en cuatro países latinoamericanos. Ellos fueron indicados por los coordinadores de las maestrías como personas representativas de cada propuesta formativa estudiada, que fueron las siguientes:

- La Maestría en Recreación y Administración del Tiempo Libre se imparte por la Universidad Regional Miguel Hidalgo desde 1997. Esta universidad está ubicada en Ciudad Madero, en México. Esta maestría es la más antigua entre las cinco estudiadas.

- La Maestría en Recreación, de la Universidad YMCA, fue creada en el año 2004 en la Ciudad de México. Esta maestría ofrece dos especialidades sobre la temática: Recreación Laboral y Recreación Turística.

- La Maestría Profesional en Recreación fue creada el año 2004 en la Universidad de Costa Rica. La primera generación empezó en el año 2005 y esta es la única maestría desarrollada en América Central sobre esta temática. 
- La Maestría en Recreación y Tiempo Libre se imparte por la Escuela Politécnica del Ejército, en Quito, Ecuador, desde el año 2006. El año 1972 esta institución formativa fue abierta a estudiantes civiles.

• La Maestría en Estudios del Ocio (“lazer” en portugués) se imparte en Brasil por la Universidad Federal de Minas Gerais y su primera generación empezó en 2007. Actualmente, desarrolla también el Doctorado Interdisciplinario en Estudios del Ocio.

Para nominar a los voluntarios que fueron entrevistados en esta investigación fue adoptada la siguiente estrategia: Cada entrevistado fue nombrado por un código iniciado por la letra E. (que corresponde a la entrevista), seguida de la situación de cada persona con la maestría entre las opciones C. (para coordinadores), P1. o P2. (profesores), E. (estudiantes) y EG. (egresados). Al final del código, se indica el país de la maestría, siendo B. de Brasil, E. de Ecuador, CR. de Costa Rica, M1. de México/URMH y M2. de México/YMCA. Las entrevistas fueron realizadas solamente después de la aprobación del Comité de Ética de la UFMG, universidad responsable por la investigación y que acompañó el proceso de estudio.

A continuación serán presentados algunos de los resultados del estudio realizado. Procurando dialogar con los marcos teóricos que fundamentaron las reflexiones sobre el papel del ocio y de la recreación en las sociedades latinoamericanas actuales, conforme fue destacado anteriormente, se aclara que Follari (2004) fue adoptado en este texto como uno de los principales referenciales teóricos que fundamentaron los análisis sobre la temática aquí investigada.

\section{El ocio y la recreación en América Latina actual}

Como plantea Escobar (2000), para hablar de América Latina es necesario considerar los lugares y realidades locales, obviamente sin perder de vista el contexto más amplio. De este modo, al considerar el contexto actual, los entrevistados fueron invitados a reflexionar sobre la pregunta clave que originó algunas de las reflexiones de este artículo. En cuanto algunos entrevistados consideran que el ocio y la recreación ocupan un lugar protagónico en el contexto latinoamericano actual, otros entienden que no son suficientemente valorizados, siendo presentada una multiplicidad de ideas para aclarar cada punto de vista.

Uno de los entrevistados resaltó que, cada vez más, el ocio se reduce a la TV, a internet y a los juegos electrónicos, como partes integrantes de una lógica consumista:

Creo que lamentablemente por las dinámicas de vida que llevamos, por el querer tener, tener, tener, tener, por la parte económica no le damos la importancia. [...] ¿Cuáles son nuestras actividades de ocio que tenemos con nuestros hijos? ¿Hacia dónde nos encaminamos si 
estamos cansados, si estamos aburridos? Estamos teniendo a los chicos viendo la televisión únicamente, o el Internet, o Nintendo y juegos electrónicos, entonces. (E.EG.M2.)

Siguiendo a Follari (2004), vemos como el consumismo a través de una pantalla es expresión de la ideología dominante en la actualidad, ya que asistimos a una época:

[...] donde triunfa la virtualidad, y hay un exceso de simbolización en relación a la condición de mundo real. Universo donde la hipertrofia de lo cultural es el signo dominante, en tanto la cultura se ha enraizado en la economía política concreta (formando parte del proceso infraestructural), a la vez que ha densificado su presencia en la vida cotidiana de los sujetos, saturándolos de mensajes de todo tipo, y enclavándolos de ese modo dentro del proceso del consumo (el que se constituye como ideología hegemónica de nuestro tiempo, pues ya no requiere sostenerse en posiciones valorativas ajenas al consumismo como tal). (Follari, 2004: 23)

En esa dirección, otro entrevistado destacó la influencia que la cultura mediática ejerce en la vida cotidiana de la población, apelando a signos y símbolos que acaban moldeando comportamientos. En este sentido, afirmó que aunque el ocio sea enfatizado por los medios de comunicación como algo importante, en los momentos de decisión las personas acaban priorizando lo que es necesario y útil en menoscabo de alguna opción que podría proporcionar un sentido más interesante, como un viaje de vacaciones. Esto acontece porque, en muchos países de Latinoamérica, la mayoría de las personas vive en situación de significativa pobreza material, lo que acaba consolidando la idea de que muchas de las posibilidades de vivir el ocio y la recreación son un lujo. Así, en el ejercicio cotidiano de priorizar lo que es más importante, muchas veces el ocio y la recreación son tratados como superfluos, secundarios o no tan necesarios.

Aunque hoy los medios de comunicación te dicen lo importante de jugar con la familia, estamos tratando o están tratando los medios y los gobiernos en general de recuperar las instancias básicas de las diferentes sociedades. [...] Pero cuando piensan entre pagar un coche o irse de vacaciones terriblemente apretados, piensan en el coche más que en las vacaciones, cuando el sentido de las vacaciones sería mayor. (E.P2.M2.)

Otro elemento importante a ser considerado en este contexto es la valorización del placer. La búsqueda de disfrute y placer puede ser satisfecha por medio de una infinidad de opciones, entre las cuales las fiestas son un campo fecundo para el imaginario social. Considerando esta posibilidad, dos entrevistados mexicanos entienden que el ocio y la recreación poseen un protagonismo en las sociedades latinoamericanas actuales debido a la importancia que los momentos festivos adquieren en la región. 
Es un papel protagónico desde el sentido de la fiesta, por ejemplo, en el sentido recreativo que tiene la fiesta en Latinoamérica está muy presente y es identificado por cualquier persona en estos países latinoamericanos. Yo digo que particularmente aquí en México, una manifestación muy tácita de lo que es la recreación son las festividades populares y las que uno mismo va adquiriendo desde otras culturas y que las vamos haciendo como parte también de nuestras prácticas. (E.E.M2.)

En el sentido de la propia vida cotidiana en que nosotros nos involucramos, yo considero que somos recreativos, lúdicos, festivos, somos los latinos. Eso creo que viene mucho por nuestra propia naturaleza y nuestra propia herencia cultural. [...] Entonces somos unos países inmensamente ricos en actividades de carácter lúdico cultural, festivo, artístico. Yo diría que la vida cotidiana de los latinos se ha hecho sin la necesidad de los especialistas y que seguramente seguirá siendo tan rica como es. (E.P1.M1.)

Ciertamente las fiestas son valorizadas en las culturas latinoamericanas como parte de construcciones culturales que destacan la relevancia de poder vivir una experiencia extraordinaria. Es, por eso, un campo fecundo para "un mundo fabricado por la representación y por el simulacro", como destaca Follari (2004: 47). Ser festivo se trata, por lo tanto, de algo culturalmente aprendido, asimilado e incorporado. En otras palabras, los latinoamericanos no son naturalmente festivos ya que esto es parte de un proceso de aprendizaje cultural que interfiere en las subjetividades y moldea los comportamientos.

Siguiendo otros parámetros, la opinión de entrevistados de distintos países partió de la prerrogativa de que la importancia y la necesidad del ocio y de la recreación están relacionadas con la salud, con la calidad de vida y con el desarrollo humano.

Entonces [...] introducimos elementos de recreación y los damos a conocer a la gente para que empiecen ya también a trabajar [...] y preguntarse cuál es su vinculación con la parte del desarrollo humano y como impacta en la calidad de vida. (E.C.M2.)

Yo tengo mucho la visión de la recreación como enfoque hacia la salud mental y salud física. Porque a nivel también como para relajarse, para integración, para tolerancia, aceptación. [...] Entonces pienso que va como por ese lado. Va hacia un enfoque de salud integral. (E.E.CR.)

Mucha gente busca, necesita actividades que le ayuden a mejorar su calidad de vida. Entonces está tratando de fomentar mucho en diferentes edades que la población realice actividades, se preocupe por su bien estar [...]. (E.EG.CR.) 
Estas consideraciones evidencian, primordialmente, la preocupación con el bienestar y con la calidad de vida de las personas, que desde la perspectiva de los entrevistados podría ser propiciado por medio del ocio y de la recreación. Aunque la expresión calidad de vida sea polisémica, en general es utilizada para indicar un estado de satisfacción, lo que tiene un fuerte componente subjetivo porque envuelve una percepción personal sobre la propia vida. Aún cuando la dimensión subjetiva sea relevante, obviamente la calidad de vida también precisa ser pensada tomando en cuenta las dimensiones objetivas, esto es, materiales, que definen la existencia. En caso contrario, se corre el riesgo de que sea reducida a un simple cliché. Por eso, Guimarães y Martins (2004) señalan que muchas investigaciones sobre el tema están relacionadas con la cuestión de la pobreza material, de la exclusión y de la desigualdad social. Desde esta mirada es necesario considerar la multiplicidad de factores involucrados de forma más amplia. Así, se habla de calidad de vida cuando se discuten los servicios y equipamientos que una ciudad o provincia entrega a sus habitantes. Se relaciona la temática con la vida saludable, calidad de alimentación y nutrición, acceso a ciertos bienes y servicios, o incluso, a espacios y productos destinados al ocio, al turismo o al consumo de bienes culturales.

De esa forma, la calidad de vida no puede ser tratada como si fuese, exclusivamente, la consecuencia de una decisión personal y subjetiva, ya que el acceso a una vida buena está determinada por una amplia gama de elementos. Recordemos que, en general, las sociedades humanas a nivel global están marcadas por fuertes formas de exclusión, discriminación, segregaciones e injusticias sociales, que determinan fuertemente el acceso a los bienes socialmente producidos y con esto, que generan imposibilidad de acceso a calidad de vida sobre el umbral de una línea de dignidad. Así, tenemos amplios sectores humanos viviendo diversos tipos de pobrezas, materiales y otras.

A su vez, se considera que el ocio y la recreación no pueden ser abordados como si fuesen los elementos centrales de la calidad de vida. A pesar de ambos ser importantes en la búsqueda de una vida con más sentido y calidad, paralelamente existen otros factores determinantes de ese proceso tales como las condiciones históricas, políticas, socioeconómicas, culturales y educacionales de los sujetos en los contextos en que viven, así como las estructuras socio-político-económicas vigentes en cada contexto social específico.

Por lo anterior se percibe como, muchas veces, los discursos sobre la calidad de vida son vaciados y banalizados, lo que es común en los tiempos de desfundamentación de hoy, en los cuales se resalta lo que Follari (2004) denomina de “metafísica del pensamiento des-situado”. O sea, aunque sean considerados problemas reales, su supuesta solución se basa en situaciones ideales.

Otro grupo de entrevistados también vinculó al ocio y a la recreación con la mejoría de la calidad de vida. Las reflexiones son desarrolladas en el 
sentido de considerar al ocio y a la recreación como derechos, teniendo presente que esta forma de entenderlos aún no está muy difundida.

El ocio es un derecho del ser humano y [...] está involucrado en mejorar su calidad de vida. Entonces nosotros podemos palpar, al menos ya en mí país puedo palpar que eso no está siendo cumplido y por varios factores, hablemos por desconocimiento de ese derecho, hablemos por las condiciones sociales de las personas. (E.EG.E.)

[...] la recreación es un derecho. Es un derecho humano y deberíamos de ejercerlo. Pero no se ejerce porque no se tiene conciencia de que lo es. (E.P1.CR.)

Yo entiendo al ocio como un derecho de las personas. [...] yo considero que es imposible pensar en una calidad de vida en la cual el ocio no sea una cuestión importante a ser tratada. (E.E.B.)

Yo pienso que la recreación y el ocio tienen un papel fundamental en la medida en que son algo que todo el mundo quiere, que todo el mundo precisa. [...] Es algo que el ser humano está buscando y a partir de esta búsqueda se constituye hoy [el ocio] como un derecho social. (E.C.B.)

Yo creo que el lugar es poder garantizar los derechos, y con esto no digo solo desde el punto de vista económico, sino también desde el punto de vista del conocimiento. A veces una persona puede tener condiciones de tener acceso al ocio y a la recreación, pero ella no lo hace, porque desconoce las posibilidades existentes [...]. (E.P1.B.)

Estas consideraciones evidencian la importancia de reconocer al ocio y a la recreación como derechos sociales. Este aspecto se torna relevante principalmente en los contextos donde las desigualdades sociales son alarmantes y revelan niveles absurdos de iniquidades, como es común en casi toda América Latina. De este modo, su realización significa algo profundo que envuelve varios elementos, los cuales posibilitan el surgimiento de interrelaciones dinámicas. Entre estos elementos pueden ser citados el reconocimiento de identidades/diversidades culturales, la creatividad social y tantos otros, que, en conjunto, pueden abrir algunas puertas para la transformación social.

Los comentarios de los entrevistados hasta ahora presentados evidencian que según ellos la recreación yel ocio poseen un protagonismo en el contexto latinoamericano. En contrapartida, varios entrevistados comparten la opinión de que este protagonismo no es tal y que solo ocupan un papel secundario -porque la sociedad, en general, no reconoce el valor del ocio y de la recreación-, lo que resalta la importancia de la educación para vivenciarlos con más autonomía.

[...] nos falta educación para el uso del tiempo libre. [...] porque la 
recreación tiene que verse como parte del estilo de vida de la gente y eso es lo que no hemos entendido, que es una de las condiciones para tener calidad de vida. (E.P1.CR.)

Lo que yo creo que hace falta es que la gente entienda que la recreación es tan importante como comer, tomar agua y andar todo el día. [...] Yo creo que lo que hace falta es enseñar más a la gente que tienen que recrearse más, que es muy importante, que es parte de la calidad de vida. (E.C.CR.)

Puedo decir que falta mucho, es decir ocupa todavía un papel secundario o terciario dentro de las prioridades de lo que tienen que hacer las personas, a pesar de que en su ocio ellos pueden ser ellos mismos, disfrutarse y disfrutar a los demás, disfrutar lo que les rodea, su cultura, pero todavía no creo que haya una conciencia plena de la importancia del ocio para el desarrollo de las sociedades. (E.P2.E.)

En los dos primeros relatos anteriores es posible verificar que permanece la vinculación del ocio y de la recreación con la calidad de vida. Además de esto, los tres entrevistados enfatizaron la importancia de la educación para que las personas reconozcan su valor y relevancia en la vida cotidiana. En la contemporaneidad, ¿¿Qué es lo que define, por un lado, la valorización del ocio y de la recreación y, por otro lado, su completa depreciación?

Las anteriores opiniones evidencian la presencia de la huella del tiempo posmoderno, que ha podido revalorizar el ocio frente al predominio de la disciplina y del trabajo que han sido propios de las llamadas sociedades modernas. Siguiendo esa línea de interpretación, algunos entrevistados entienden que la desvalorización del ocio y de la recreación es consecuencia de la prioridad atribuida al trabajo en detrimento de todo aquello que es considerado inútil o improductivo.

[...] en la actualidad las personas trabajan y [...] ya no tienen tiempo para recrearse, ni para el ocio. Están todo el tiempo trabajando, produciendo. (E.E.E.)

Creo que tiene un papel secundario. Para la gente es más importante el trabajar y después de trabajar la función de ser padre, los que son padres o familia, y en último lugar queda la cuestión de la recreación. (E.P2.M1.)

Bueno, yo considero que en un mundo globalizado como en el que vivimos [...] la recreación y el tiempo libre están teniendo cada vez menos espacios. A pesar de que es un área de la investigación y del conocimiento y de la vida muy importante, no le estamos dando el lugar que merece. Yo pienso que están en segundo plano luego de la parte netamente laboral, técnica, profesional a la que las personas se dedican. (E.P1.E.) 
Desde aquí es posible verificar que, a menos que la recreación y el ocio sean imprescindibles para impulsar el consumo masivo de bienes y de servicios, son relegados a un según plano cuando el énfasis al trabajo y a la lógica capitalista son lo más importante. ¿Qué lugar ellos pasan a ocupar, entonces, en este contexto? Según lo que fue relatado por un entrevistado:

[...] todo el mundo está tenso, estresado y a veces yo visibilizo la recreación como una medicina. Cuando tenemos dolor de cabeza nos tomamos una pastilla. Se nos quitó el dolor de cabeza y ya no tomamos ni una. Si no nos duele la cabeza ya no la tomamos. Entonces si estoy tensa voy y hago un poquito de deporte o me río, hago algo de recreación. Pero si no estoy tensa, no lo busco. (E.P1.CR.)

Como es indicado en el anterior comentario, muchas veces el ocio y la recreación son visualizados por las personas como un remedio para combatir algunos problemas, como el estrés y la tensión. De esa forma, recreación y ocio podrían ser vistos como simulacros, esto es, como posibilidades encargadas de solucionar algún problema -aunque eso sea sólo aparentemente, por la vía de la simulación-.

Según Bistué (2004), es preciso rebatir y desenmascarar las ilusiones salvacionistas y las abstracciones, recuperando la responsabilidad humana de luchar para construir y consolidar un mundo "más vivible”. Riesman (1971) complementa esa idea al esclarecer que el ocio no es capaz de salvar el trabajo, pues, fracasa junto con él. De ese modo, el autor entiende que el ocio será significativo para las personas cuando el trabajo también lo sea.

De acuerdo con Pinto (2009), considerar al ocio y a la recreación como apéndices del trabajo productivo es una manera utilitarista de entenderlos. Esa visión fue cuestionada por dos estudiantes mexicanos:

[...] para mí el ocio no tiene que ser tanto utilitario. Realmente no debe ser utilitario pero a la larga es utilitario. A la larga es utilitario en tu vida porque te vas, conversas, etc., pero llegas a tu tiempo serio de trabajo y ves que eso sí fue útil y que puedes rendir un poco más. (E.E.M1.)

Entonces la recreación [...] se vuelve como un paliativo, es decir, como algo que nos distrae de muchas problemáticas. Pero también puede nos ayudar a sanar sobre lo que nos causa esa problemática. Ahí encontramos la oportunidad de expresar nuestras emociones, nuestros sentimientos o nuestras frustraciones y quizá la oportunidad también de demostrar que tenemos esperanza de seguir queriendo ser mejores y superar tales problemáticas. (E.E.M2.)

En este artículo se postula que no hay fronteras absolutas y rígidas entre trabajo y ocio, tampoco entre el ocio y las obligaciones cotidianas. Aunque el sujeto esté buscando satisfacción, más flexibilidad y libertad de elección a través del ocio, no siempre estará libre de obligaciones sociales, 
familiares, profesionales, institucionales, etc. Estos aspectos se mezclan e influyen, dinámicamente, en la vida cotidiana (Gomes, 2004).

A su vez, los relatos anteriores pueden ser complementados con el punto de vista de otro entrevistado, cuando este llama la atención sobre a importancia del ocio para cuestionar la perspectiva instrumental que marca el trabajo y también la vida cotidiana como un todo.

Nosotros tenemos una tradición muy fuerte de mirar para el mundo por la perspectiva de la razón instrumental y el ocio toca en esta herida, el ocio nos provoca. [...] nuestra historia humana se construyo por la dimensión del pensamiento, y la gente olvidó lo que nosotros, los seres humanos, vivimos. De la estética del cuerpo en cuanto producción humana. Entonces el ocio toca en esta historia. La otra dimensión que es marcadora es la dimensión del trabajo. Nosotros construimos una mirada ética sobre el mundo por la lógica del trabajo, pero no por el trabajo como noción de práxis o por esta relación práxis y poiesis [...], y si por el trabajo como hacer objetivo, como hacer, como consecuencia lineal: yo hago esto y produzco eso. O sea, el mundo que se ve como instrumentalidad no dialógica, no complejo, como causa y efecto [...]. Entonces, esta lógica del trabajo también precisa ser tensionada por el ocio. (E.P2.B.)

En las sociedades capitalistas neoliberales, la forma más inmediata de abordar la temática del ocio expresa su vinculación con el trabajo, lo que es comprensible porque el aspecto relacional pone en evidencia las fronteras existentes entre ambos. Pero esto es comprensible hasta cierto punto ya que lo más común es la reducción del ocio a una relación dicotómica donde su existencia es justificada solamente por las necesidades e intereses del trabajo productivo, lo que representa uno de los principales aspectos del concepto moderno de ocio.

Toda relación dicotómica combina la simetría con la jerarquía, como aclara De Sousa Santos (2002). De esta forma, la simetría entre las partes es siempre una relación horizontal que oculta una relación vertical. Esto sugiere que ninguna de las partes tenga vida propia más allá de la que es atribuida por la relación dicotómica. En esta perspectiva, el autor propone repensar los términos de las dicotomías fuera de las articulaciones y relaciones de poder que unen cada parte como un primer paso para liberarlas y ampliarlas. Esto también puede servir para revelar otras alternativas que quedan desconsideradas por las dicotomías hegemónicas.

Para superar jerarquías, relaciones de poder y dicotomías, es esencial pensar al ocio y a la recreación como si no hubiese trabajo, por ejemplo. Esto no significa eliminar el aspecto relacional que los acerca, pero pone en relieve a algunas preguntas fundamentales: ¿Qué es lo que existe en el ocio que escapa a la dicotomía trabajo/ocio?¿El ocio puede ser una totalidad, o solamente puede ser comprendido como algo vinculado al trabajo? 
Por lo anterior, es relevante recordar que los pueblos y sociedades que componen la región latinoamericana construyen y significan sus prácticas socioculturales, que son vividas como disfrute de la cultura y de la vida social en sus propias temporalidades. Así, el ocio no siempre está, ni estuvo subyugado al trabajo, a la urbanización industrial, tampoco al capitalismo neoliberal, a pesar de estar en el presente en íntima relación con estos y con otros elementos del contexto actual.

En la vida cotidiana, el ocio constituye relaciones dialógicas con otros campos además del trabajo, tales como la educación, la política, la economía, el lenguaje, la salud, el arte, la ciencia y la naturaleza, entre otras dimensiones de la vida, siendo parte integrante y constitutiva de cada sociedad. Por lo tanto, el ocio es constituido conforme a las peculiaridades del contexto en el cual es desarrollado implica una producción de cultura -en el sentido de ser reproducción, construcción y transformación de prácticas culturales vivenciadas lúdicamente por personas, grupos, sociedades e instituciones. Estas acciones son construidas en un tiempo/espacio social, dialogan y sufren interferencias de las demás esferas de la vida en sociedad y nos permiten resignificar, simbólica y continuamente, la cultura (Gomes, 2008). Este es uno de los aspectos importantes para comprender el ocio "postmoderno".

Sumado a lo anterior se destaca que, en esta investigación, algunas personas buscaron reflexionar sobre el papel del ocio y de la recreación en las sociedades latinoamericanas actuales vinculándolos con la ética del trabajo y también con las problemáticas sociales y políticas más amplias que marcan la región. Además, tres entrevistados procuraron aproximarse a estos aspectos para contextualizar sus puntos de vista, como ilustran los ejemplos a continuación.

[...] Entonces yo me imagino que como somos Estados latinoamericanos [...] debemos de estar inmersos en lo mismo. Por lo poco que se ve somos igualitos en las condiciones de países en vía de desarrollo, de pobreza, me imagino que debe de tener situaciones iguales de recreación. (E.EG.E.)

Pues yo considero que debería de ser un lugar preponderante, debería de ocupar uno de los primeros lugares. Pero considero que no se encuentra en ese punto que debería de estar. Porque la misma situación económica, la dinámica de los países de América Latina, que es más si estás, por ejemplo, laborando, buscando el aspecto económico. (E.P1.M2.)

[...]si nosotros conseguimos hacer que las personas entiendan que el ocio está muy ligado a la humanización, muy ligado al propio sujeto, al relacionarse mejor consigo mismo y con la sociedad, que el ocio no es solamente una válvula de escape [...]. Todo esto puede hacer que la calidad de vida en América Latina como un todo se mejore. Que las personas se tornen más conscientes de su papel 
social, de lo que ellas representan y así la vida pueda tener más sentido. (E.EG.B.)

Estos comentarios indican que la formación de profesionales para el campo de la recreación, el tiempo libre y el ocio necesita contemplar el desafío de pensar críticamente en el sentido de buscar alternativas para las graves problemáticas presentes en las sociedades latinoamericanas, algo que es cada vez más urgente y necesario. Al final,

[...] ¿De qué vale el pacto social cuando hay quienes no están socialmente integrados, y por tanto son obviamente ajenos a las condiciones del pacto? ¿Sobre qué situación ideal de habla podríamos pensar seriamente en la Latinoamérica actual conformada por desposeídos, excluidos, desocupados, que no hablan -de lo público- siquiera en condiciones no ideales, dado que están fuera del juego político explícito? Por supuesto que lo dicho no significa que debamos abstenernos de pensar hasta esperar que la situación de los excluidos esté solucionada. Por el contrario: hay que pensar para que algún día pueda solucionarse. (Follari, 2004: 22)

Como aclara Bistué (2004), muchas veces se considera que el pensamiento crítico está detenido por motivos supuestamente internos al propio pensamiento. Para la autora eso sería una conclusión miope, pues, la pretensión de explicar los avatares del pensamiento crítico como resultado del movimiento inmanente al acto de pensar, como si él fuese una idea abstracta, es un equívoco. En esa perspectiva, es preciso colocar al pensamiento dentro de una totalidad más abarcadora que lo haga inteligible. Para eso, es necesario buscar los elementos materiales que hicieron posible esta deriva a nivel de pensamiento. "Hoy resulta clave poner en conexión la detectada recesión del pensamiento crítico con sus bases materiales a fin de avanzar en la comprensión de nuestra contemporaneidad y sus posibilidades de transformación.” (Bistué, 2004: 54)

Siguiendo esta línea de interpretación, es preciso tomar conciencia que las acciones como profesionales y como investigadores del ocio y de la recreación no son neutras: ellas son siempre sociales y políticas, dejan huellas y tienen variados efectos y repercusiones. Una persona puede ser experta en el desarrollo técnico de muchas prácticas recreativas, pero esto es insuficiente si no es considerada la necesidad de desarrollar una reflexión sistematizada sobre el sentido de estas mismas prácticas. Además, es preciso superar la condición de actuar, exclusivamente, como receptores del conocimiento producido en otros contextos, muchas veces reproduciendo, sin darse cuenta, la lógica capitalista de producción/consumo. Es fundamental, así, generar conocimientos pertinentes, contextualizados y críticos sobre la temática (Gomes, 2011).

Es necesario destacar que muchos estudios desarrollados sobre la recreación en Latinoamérica buscan ser un aporte para el desarrollo humano a partir de una postura crítica, cuestionadora y comprometida con el 
alcance de una vida buena para todos. A su vez, existen estudiosos e instituciones latinoamericanas que valorizan el potencial lúdico y creativo de la recreación, ya sea como una herramienta educativa o como una posibilidad de animación sociocultural, campo de actuación de la educación física, del turismo, de la psicología social, de la pedagogía, del trabajo social, entre otras áreas. (Gomes; Elizalde, 2012)

Por eso, cada vez más los estudios e intervenciones en el campo del ocio y de la recreación precisan considerar la búsqueda de alternativas para enfrentar situaciones complejas como las desigualdades sociales, la precarización del trabajo, el descuido de los derechos sociales, los conflictos armados, la marginalización y las distintas formas de violencia que marcan profundamente toda la región latinoamericana, entre muchas otras. Al final, existe una estrecha relación entre las formas de organizar el conocimiento a nivel mundial y las estructuras económicas y de poder que son hegemónicas.

En ese sentido, una interesante reflexión fue hecha por unos de los profesores entrevistados en la investigación:

Todavía estamos quizá trasplantando modelos, modelos de lo que es recreación y deberíamos de fortalecer más los procesos de recreación desde nuestro contexto, desde el contexto del barrio, el contexto del lugar, del pueblito. Porque de acuerdo a cada contexto hay una forma distinta en la que se puede aprovechar el rato de ocio. No podemos hablar con un mismo discurso, un discurso urbano de clase media alta para toda la población. Tenemos que contextualizar más estos discursos. (E.P2.CR.)

Además, las perspectivas contextualizadas sobre la temática del ocio precisan ser construidas tomando en consideración las prácticas socioculturales y los saberes que vienen siendo elaborados en los territorios latinoamericanos, considerando:

[...] las culturas de los pueblos indígenas, de los grupos descendientes de África y de otros continentes del mundo, que son la base de la diversidad cultural y étnica de la región; las teorías históricas, sociales, ecológicas, (geo)políticas y pedagógicas que marcan el pensamiento crítico de autores latinoamericanos; la educación popular y los movimientos de base; las prácticas comunitarias de autogestión, la economía solidaria, bien como tantas otras prácticas y experiencias alternativas al sistema neoliberal capitalista desarrolladas con éxito en el contexto latinoamericano, a pesar de ser poco conocidas. Lo importante es que muchas de estas prácticas están siendo desarrolladas en América Latina como alternativas concretas para superar las problemáticas existentes en esta región. (Gomes; Elizalde, 2012: 248)

Teniendo en vista la importancia de enfrentar estos y otros desafíos 
para los estudios del tema, el ocio -histórica, cultural y socialmente situado, problematizador, crítico, sinérgico y transformacional- puede ser una significativa herramienta para movilizar experiencias interculturales y educativas contrahegemónicas (Elizalde, 2010), contribuyendo así con un aprendizaje para la transformación social y cultural.

\section{Consideraciones finales}

Como fue tratado en este artículo, resulta necesario pensar la posmodernidad vinculándola con el ocio y la recreación ya que ellos pueden contribuir, y de hecho lo hacen, con la formación de las subjetividades en distintos contextos culturales y sociales. En los últimos años, esto es cada vez más influenciado por el avance tecnológico, por la difusión de los medios masivos de comunicación y por el consumo compulsivo, entre otros elementos que acaban moldeando comportamientos y configurando nuevas prácticas sociales.

En relación a los valores hegemónicos en el contexto actual, fue constatado en este estudio que el ocio y la recreación son ambiguos: algunas veces son considerados importantes para promocionar el bienestar y la calidad de vida de las personas, pero, otras veces, son vistos como superfluos y desnecesarios ya que la ética del trabajo continua siendo muy asimilada en las sociedades latinoamericanas actuales, como fue indicado por algunos entrevistados.

Sabemos que el ocio y la recreación pueden contribuir, y de hecho lo hacen, con el mantenimiento del status quo y del orden social injusto, reforzando estereotipos y valores excluyentes, consumistas y alienantes. Pero a su vez, paradojalmente, también pueden abrir posibilidades para la construcción de sociedades humanas, inclusivas, participativas y solidarias. Todo esto revela que, en nuestras sociedades, el ocio es un fenómeno dinámico, complejo, dialógico y lleno de conflictos, tensiones, ambigüedades y potencialidades (Gomes; Elizalde, 2012).

Desde este punto de vista, uno de los desafíos pendientes para este campo de estudios es profundizar las reflexiones sobre estas temáticas buscando la superación de la idea de que el valor del ocio y la recreación están subyugados al trabajo: sea proporcionando diversión, descanso, compensación y evasión por medio de los juegos electrónicos y de la virtualidad, de los viajes, de la fiesta y de otras experiencias recreativas, como fue destacado por distintos entrevistados en este estudio. Desde aquí es posible verificar que, a menos que el ocio y la recreación sean imprescindibles para impulsar el consumo masivo de bienes y de servicios, son relegados a un según plano cuando el énfasis al trabajo y a la lógica capitalista son lo más importante.

En este sentido, necesita ser revisada la forma habitual de conceptualizar al ocio y a la recreación principalmente en función del traba- 
jo. Este aspecto atravesó, de modo transversal, distintas entrevistas y junto con su vinculación a la calidad de vida, fueron las dos dimensiones más significativas en relación a la comprensión del papel del ocio y de la recreación en las sociedades latinoamericanas actuales.

En suma, según los marcos teóricos, las percepciones de los entrevistados y los análisis realizados en este artículo, el ocio y la recreación necesitan ser conceptualizados y resignificados teniendo como base otras miradas que contengan la semilla de la reflexión sobre los propios hábitos y costumbres vividos cotidianamente. De este modo, las personas interesadas en profundizar conocimientos sobre las temáticas estudiadas en esta investigación pueden colaborar con este proceso de preguntarnos y abrir otras miradas sobre el mundo social y el ser humano. 
Polis, Revista Latinoamericana, Volumen 13, $N^{\circ}$ 37, 2014

\section{Notas}

${ }^{1}$ Docente de la Universidade Federal de Minas Gerais, Brasil (UFMG). Líder del Grupo de investigación Otium: Lazer, Brasil \& América Latina (UFMG/CNPq). Email:chrislucegomes@gmail.com

${ }^{2}$ Este artículo es fruto del proyecto de Post-doctorado titulado "Lazer/Ocio e Recreação/ Recreación na América Latina Atual: Análise dos Mestrados de Brasil, Costa Rica, Equador e México”, que integró una investigación más amplia, realizada con el apoyo de CNPq, FAPEMIG y Ministerio del Deporte/Brasil. El Post-doctorado fue desarrollado en la Facultad de Ciencias Políticas y Sociales de la Universidad Nacional de Cuyo (2011-2012), bajo la dirección del profesor Dr. Roberto Follari, a quien agradezco por las contribuciones a este texto. Agradezco también al profesor Dr. Rodrigo Elizalde por la revisión y por los aportes a este artículo. 


\section{Bibliografía}

Bauman, Z. (2001), Modernidade Líquida, Jorge Zahar Editor, São Paulo.

Bistué, N. (2004), “El trabajo de lo negativo. Avatares del pensamiento crítico”. En: Follari, R.; Bistué, N.; Yarza, C. (Org.). La proliferación de los signos: La teoría social en tiempos de globalización, Homo Sapiens, Rosario pp.53-78.

Bittar, E. C. B. (2008), “O direito na pós-modernidade”. Revista Sequência $\mathrm{n}^{\circ} 57, \mathrm{p} .131-152$.

Elizalde, R. (2010),“Resignificación del ocio: Aportes para un aprendizaje transformacional”. En: Revista Polis, V. 9. N 25. Universidad Bolivariana, Santiago pp. 437-460. Disponible en: <http://www.scielo.cl/pdf/polis/ v9n25/art26.pdf> Acceso el: 25 marzo 2012.

Follari, R. (1990), Modernidad y Posmodernidad. Una óptica desde América Latina, Aique/Reí/IDEAS, Buenos Aires.

Ídem (2004), “El auge de la Filosofía Política como síntoma”. En: Follari, R. (coord.); Bistué, N.; Yarza, C. La proliferación de los signos: La teoría social en tiempos de globalización, Homo Sapiens, Rosario pp.15-28.

Follari, R.; Lanz, R. (coord.) (1998), Enfoques sobre posmodernidad en América Latina, Editora Sentido, Caracas.

Gomes, C.L. (2011),“Estudos do Lazer e geopolítica do conhecimento”. En: Revista Licere V.14, N³, Belo Horizonte, pp.1-25, set./2011. Disponible en <http://www.anima.eefd.ufrj.br/licere/sumario.html?ed=29> Acceso el 27 mar. 2012.

Ídem (2008) Lazer, trabalho e educação: relações históricas, questões contemporâneas. 2. ed. rev. ampl. Editora UFMG, Belo Horizonte.

Ídem (2004) Lazer - concepções. In: ___ (Org.). Dicionário crítico do lazer. Editora Autêntica, Belo Horizonte, p. 119-126.

Gomes, C.L.; Elizalde, R. (2012), Horizontes latino-americanos do lazer/ Horizontes latinoamericanos del ocio, Editora UFMG, Belo Horizonte. Disponible en <http:/grupootium.files.wordpress.com/2012/06/ horizontes_latino_americanos_lazer_junho_20123.pdf> Acceso el: 27 sep. 2012.

Guimarães, E.; Martins, V.L.A.B. (2004), “Qualidade de vida”. En: Gomes, C.L. (coord.), Dicionário crítico do lazer, Autêntica Editora, Belo Horizonte, pp.191-196.

Hall, S. (2005), A identidade cultural na pós-modernidade, DP\&A, Rio de Janeiro. 
Harvey, D. (1993), Condição pós-moderna, Loyola, São Paulo.

Jameson, F. (1999), El giro cultural, Manantial, Buenos Aires.

Laville, C.; Dionne, J. (1999), A construção do saber: Manual de metodologia da pesquisa em ciências humanas. Artmed, Porto Alegre.

Lipovetsky, G. (2007), Felicidade paradoxal: ensaio sobre a sociedade de hiperconsumo, Companhia das Letras, São Paulo.

Pinto, G.B. (2009), O lazer em hospitais: Realidades e desafios, UFMG, Belo Horizonte (Dissertación, Maestría en Ocio/Lazer).

Riesman, D. (1971), A multidão solitária, Perspectiva, São Paulo.

Sennet, R. (2006), A Corrosão do Caráter: as conseqüências pessoais do trabalho no novo capitalismo, Record, Rio de Janeiro.

Sousa Santos, B. (2002), "Para uma sociologia das ausências e uma sociologia das emergências”. Revista Crítica de Ciências Sociais 63, Outubro, p. 237280, 2002.

Ídem (2011), A critica da razão indolente: Contra o desperdício da experiência. Editora Cortez, São Paulo.

Recibido: 06.12.2012

Aceptado: 19.06.13 